\title{
Predictors of upstage and treatment strategies for stage IA lung cancers after sublobar resection for adenocarcinoma in situ and minimally invasive adenocarcinoma
}

\author{
Hang Su ${ }^{1 \#}$, Chang Gu ${ }^{1 \#}$, Yunlang She ${ }^{1 \#}$, Long Xu ${ }^{1 \#}$, Ping Yang ${ }^{2}$, Huikang Xie ${ }^{3}$, Shengnan Zhao ${ }^{3}$, \\ Chunyan $\mathrm{Wu}^{3}$, Dong Xie ${ }^{1 *}$, Chang $\mathrm{Chen}^{1 *}$ \\ ${ }^{1}$ Department of Thoracic Surgery, Shanghai Pulmonary Hospital, Tongji University School of Medicine, Shanghai, China; ${ }^{2}$ Division of \\ Epidemiology, Department of Health Sciences Research, Mayo Clinic College of Medicine, Rochester, MN, USA; ${ }^{3}$ Department of Pathology, \\ Shanghai Pulmonary Hospital, Tongji University School of Medicine, Shanghai, China \\ Contributions: (I) Conception and design: H Su, C Gu, Y She, L Xu, D Xie, C Chen; (II) Administrative support: D Xie, C Chen; (III) Provision of \\ study materials or patients: D Xie, C Chen; (IV) Collection and assembly of data: P Yang, H Xie, S Zhao, C Wu; (V) Data analysis and interpretation: \\ H Su, C Gu, Y She, L Xu, P Yang, H Xie, S Zhao, C Wu; (VI) Manuscript writing: All authors; (VII) Final approval of manuscript: All authors. \\ \#These authors contributed equally to this work. \\ *These authors contributed equally as co-senior authors. \\ Correspondence to: Dong Xie; Chang Chen. Department of Thoracic Surgery, Shanghai Pulmonary Hospital, Tongji University School of Medicine, \\ Shanghai 200433, China. Email: kongduxd@163.com; chenthoracic@163.com.
}

Backgroundk Invasive adenocarcinoma intraoperatively underestimated as adenocarcinoma in situ (AIS) or minimally invasive adenocarcinoma (MIA) based on frozen section (FS) is more likely to undergo insufficient resection. We aimed to investigate the predictors of upstage and treatment strategies for stage IA invasive adenocarcinoma after sublobar resection for AIS and MIA.

Methods: We identified 2,006 patients from January 2012 to December 2016 with early-stage lung adenocarcinoma who underwent sublobar resection based on FS diagnosis to guide surgical decision-making. All FS were categorized into three groups in real-time: (I) atypical adenomatous hyperplasia (AAH), (II) AIS, and (III) MIA.

Results: A total of $272(13.5 \%, 272 / 2,006)$ cases were upstaged in the final pathology (FP) diagnosis (82 AAH to AIS, 127 AIS to MIA, and nine AIS and 54 MIA to invasive adenocarcinoma), and most upstage cases $(64.3 \%, 175 / 272)$ were attributed to sampling error. Multivariate logistic regression showed that tumor size $\geq 1 \mathrm{~cm}$ was the only independent predictor of upstage. The upstage of 209 cases to AIS or MIA had no influence on the therapy because the extent of their resection was enough. Of the 63 cases upstaged to invasive adenocarcinoma, only 13 cases agreed to receive complementary treatment: five patients underwent complementary lobectomy, and seven patients received chemotherapy. Two invasive adenocarcinoma cases without complementary treatment experienced a local recurrence after surgery. No recurrence was observed in AAH, AIS and MIA. No patient died until December 01, 2019.

Conclusions: Timely complementary treatment is encouraged in AIS/MIA upstaged to invasive adenocarcinoma based on the FP after sublobar resection to avoid local recurrence. Pathologists should be more cautious about AIS and MIA with tumor size $\geq 1 \mathrm{~cm}$ to avoid underestimation and potentially insufficient resection.

Keywords: Sublobar resection; frozen section (FS); lung adenocarcinoma

Submitted Jul 08, 2020. Accepted for publication Nov 18, 2020.

doi: $10.21037 /$ tlcr-20-828

View this article at: http://dx.doi.org/10.21037/tlcr-20-828 


\section{Introduction}

Lung cancer is the main cause of death for cancer patients, of which adenocarcinoma, accounts for nearly $50 \%$ and is the most common histological subtype $(1,2)$. The 2020 National Comprehensive Cancer Network (NCCN) guidelines recommend lobectomy and systematic evaluation of the mediastinal lymph nodes as the optimal choice for treating early-stage lung adenocarcinoma (3). With increasingly detection rate of early-stage lung adenocarcinoma, sublobar resection as a treatment option for early-stage lung adenocarcinoma is of great interest (4), and its use is increasing (5). Clinically, patients with adenocarcinoma in situ (AIS) and those with minimally invasive adenocarcinoma (MIA) will have a good overall prognosis after resection and therefore benefit from sublobar resection (6). However, patients with invasive adenocarcinoma who undergo sublobar resection have an increased risk of recurrence compared with those undergoing lobectomy (7-9).

Recently, intraoperative frozen section (FS) has been proven to have a high rate of agreement with the final pathology (FP) and the precise diagnosis based on FS could be used to guide surgical strategy for early-stage lung adenocarcinoma (10). In general, sublobar resection will be performed once the tumor was diagnosed as AIS or MIA by intraoperative FS. Although invasive adenocarcinoma can be definitively identified, some pitfalls in the FS diagnosis of AIS, MIA, and lepidic-predominant invasive adenocarcinoma exist. AIS and MIA based on FS may be upstaged to invasive adenocarcinoma by the FP due to suboptimal FS quality, sampling error or interpretative error (11-13). Consequently, this process would represent a histological upstage, and insufficient resection may be performed in these patients. To date, whether patients with invasive adenocarcinoma that has been intraoperatively underestimated as AIS or MIA and who underwent sublobar resection need further therapy, such as complementary lobectomy or postoperative chemotherapy, remains unclear.

In this study, we investigated the diagnostic accuracy of FS, the reasons for the discrepancy between FS and $\mathrm{FP}$, and the appropriate treatment strategy for AIS/MIA with histological upstage based on the FP after sublobar resection.

We present the following article in accordance with the TRIPOD reporting checklist (available at http://dx.doi. org/10.21037/tlcr-20-828).

\section{Methods}

\section{Patient selection}

The present study represents a retrospective review of patients with pulmonary nodules who underwent sublobar resection for intraoperatively FS diagnosis to guide surgical decision-making in Shanghai Pulmonary Hospital between January 2012 and December 2016. Inclusion criteria included two main parameters: (I) atypical adenomatous hyperplasia (AAH), AIS, and MIA diagnosed by intraoperatively FS and underwent sublobar resection (wedge resection or segmentectomy) and (II) timely and clear diagnosis of FS. Exclusion criteria consisted of three parameters: (I) compromised sublobar resection (patients with decreased pulmonary function or comorbid diseases, such as underlying pulmonary disease and/or heart disease, and those of advanced age who would not tolerate a lobectomy $(4,5,14)$; (II) histological downgrade based on the FP; and (III) multiple lung adenocarcinoma. The flowchart of patient selection is shown in Figure S1. All the patients were staged according to the 8th TNM staging system proposed by the American Joint Committee on Cancer (AJCC) (15). The end date of follow-up period was December 01, 2019.

All procedures performed in this study were in accordance with the Declaration of Helsinki (as revised in 2013) and approved by the Institutional Review Board of the Shanghai Pulmonary Hospital (IRB, K20-432). Because of the retrospective nature of the research, the requirement for informed consent was waived.

\section{Surgical treatment}

In our hospital, there were 2 surgical indications of sublobar resection, including intentional and compromised sublobar resection, but we only included intentional sublobar resection in the present study. For intentional sublobar resection, patients were required to meet all of the following criteria according to previous studies (16-18): (I) $<3 \mathrm{~cm}$ in size with pure ground glass nodules (GGN) or non-invasive appearance (consolidation to tumor ratio, CTR $<0.5$ ) on preoperative computed tomography (CT) scans, (II) tumors were diagnosed as AAH, AIS, or MIA by intraoperative FS, (III) center of the tumor was located within the outer third of the lung parenchyma, and (IV) general condition and pulmonary function adequate for lobectomy. As for the choice of wedge resection versus segmentectomy, 
the decision mainly relies on tumor location and the surgeon's skills. Selective dissection of the mediastinal lymph node was recommended for sublobar resection, and selective sampling was also accepted after a comprehensive evaluation (19).

During the study period, there were no guidelines for the treatment for AIS/MIA with FP upstage to stage IA invasive adenocarcinoma after sublobar resection. As for the complementary treatment (complementary lobectomy or chemotherapy), the surgeons gave fully informed consent to the patient and family members. In our hospital, complementary treatment was encouraged but not mandatory, and only in cases with the consent of the patient or the family member, would complementary treatment be performed.

\section{Intraoperative FS diagnosis}

The protocols of FS and postoperative FP diagnoses (FS diagnosis based on only one block for a quick diagnosis and FP diagnosis based on both two blocks from entire tumor). After tumor specimens resected, pathologists made immediate diagnoses. Specimens were sliced into block 1 and block 2 along the largest diameter of the tumor. Two or three levels of tissue section were taken for diagnosis at the largest diameter interface. Remaining tissues collected during surgery were fixed in $10 \%$ formaldehyde, paraffinembedded, prepared for final pathological examination.

Two senior pathologists independently reported the results of FS. When a disagreement occurred, a third senior pathologist was needed for an accurate diagnosis. Intraoperative pathology reports conformed to the international multidisciplinary classification of lung adenocarcinoma, which proposed by International Association for the Study of Lung Cancer (IASLC)/ American Thoracic Society (ATS)/European Respiratory Society (ERS) (2). The diagnostic performance of FS diagnosis was quantified by accuracy, sensitivity, specificity, positive predictive value ( $\mathrm{PPV}$ ) and negative predictive value $(\mathrm{NPV})$.

\section{Evaluation of permanent section slides}

Two senior pathologists independently reviewed all hematoxylin and eosin $(\mathrm{H} \& \mathrm{E})$ stained permanent section slides, and disputable cases were additionally reviewed by a third senior pathologist for accurate diagnoses. FP reports conformed to the IASLC/ATS/ERS multidisciplinary classification of lung adenocarcinoma. If the case was invasive adenocarcinoma, the percentage of each histological subtype was recorded in 5\% increments. Visceral pleural invasion (VPI) and tumor spread through air space (STAS) were also investigated.

\section{Determining the causes for misdiagnoses of FS diagnoses}

The results of our previous study revealed three major causes for the discrepancy between FS and FP diagnoses: (I) low-quality FS, (II) interpretation error, and (III) sampling error (13). To determine the specific reasons, all 272 cases that had been underestimated by FS were retrospectively reviewed by five senior pathologists in two steps via a multihead microscope.

\section{Step one}

All FS slides were divided into high and low-quality groups referenced to the details of FS quality evaluation standards in our previous paper (13). If low quality influenced the diagnosis, the upstage was attributed to low quality of FS. FS slides of high quality went to the next step.

\section{Step two}

Two types of interpretation errors, including objective and subjective interpretation errors. If the inflammation, interstitial fibrosis, or other interference factors caused difficulty interpreting the presence or extent of invasion component, the upstage was attributed to objective interpretation error. If the FS slides were without inflammation, or interstitial fibrosis or other interference factors, and FS diagnosis was changed to consistent with the FP diagnosis after re-evaluation by the five senior pathologists, the upstage was attributed to subjective interpretation error. In cases in which the FS slides were without inflammation or interstitial fibrosis, and FS diagnosis results were still AIS or MIA after re-evaluation, the misdiagnosis was attributed to FS sampling error.

\section{Postoperative follow-up}

All patients were followed up from the date of surgery after sublobar resection. Physical examination, blood tests (including tumor markers), chest CT scans, ultrasonography of the upper abdominal, and MRI of the brain were performed every three months in the first two years, every 6 months for three to five years, and once a year from then on. Bone scintigraphy was examined annually. Local 
recurrence defined as tumor recurrence in the ipsilateral hemithorax, including the resection margin, ipsilateral lung and pleura or the hilum and mediastinal lymph nodes. Distant recurrence was defined as tumor recurrence in the contralateral hemithorax or extrathoracic organs. Recurrence-free survival (RFS) was defined as the time from surgery until recurrence at local or distant.

\section{Statistical analysis}

Clinical characteristics and perioperative outcomes were analyzed using SPSS 26.0 software (SPSS Inc, Chicago, IL, USA). Categorical variables were compared using Pearson $\chi^{2}$ tests and continuous variables were compared using twosample $t$-tests. In addition, a logistic regression model was applied to confirm the independent predictive factors of upstage. The two-tailed significance level was set at $\mathrm{P}<0.05$.

\section{Results}

\section{Clinicopathological characteristics of invasive lung adenocarcinoma underestimated by FS}

A total of 2,006 patients who had undergone sublobar resection were recruited into our study, including $77 \mathrm{AAH}$, 1,039 AIS, 827 MIA, and 63 invasive adenocarcinomas. The clinicopathologic characteristics of the 2,006 patients are summarized in Table 1. Between the two groups, the characteristics of gender, smoking history, total lymph node removed, surgical procedure, video-assisted thoracic surgery (VATS), and STAS did not differ, and the distributions of the primary tumor location were also similar. However, tumors in the invasive adenocarcinoma group had a larger solid component size ( 0.71 versus $0.02 \mathrm{~cm} ; \mathrm{P}<0.001)$, larger whole tumor size $(1.47$ versus $0.83 \mathrm{~cm} ; \mathrm{P}<0.001)$, lager CTR $(0.48$ versus $0.02 ; \mathrm{P}<0.001)$ on the CT scan, and a higher frequency of higher preoperative CEA level $(12.7 \%$ versus $6.1 \% ; \mathrm{P}=0.035)$, VPI $(3.2 \%$ versus $0 \% ; \mathrm{P}<0.001)$ and postoperative chemotherapy $(11.1 \%$ versus $0 \% ; \mathrm{P}<0.001)$ than the AAH/AIS/MIA group.

Of the 63 invasive adenocarcinoma cases, 57 exhibited lepidic predominant, two papillary predominant, and four acinar predominant subtypes. No positive lymph node was found in these patients. The percentages of each histological subtype are shown in Figure 1, of which 63 cases contained the lepidic subtype, 42 cases contained the acinar subtype, 29 cases contained the papillary subtype, four cases contained micropapillary subtype, and one case contained solid subtype. Furthermore, two case were VPI positive and one case was STAS positive. In summary, eight cases exhibited pathological malignant behavior (micropapillary or/and solid pattern or/and VPI or/and STAS).

During the study period, a total of 2,153 patients with early-stage lung adenocarcinoma who enrolled to this intentional sublobar resection protocol, which were correctly diagnosed as invasive adenocarcinoma by FS and converted to lobectomy. The comparison of clinicopathological features were shown in Table S1 between invasive adenocarcinomas with correct diagnosis by FS and 63 invasive adenocarcinomas with underestimation by FS.

\section{The predictors of upstage in FP diagnosis}

Totally, 272 cases with pathological upstage by FP, $82 \mathrm{AAH}$ upstaged to AIS, 127 AIS upstaged to MIA, and 9 AIS and 54 MIA upstaged to invasive adenocarcinoma (Table 2). The overall concordance rate between FS and FP diagnoses was $86.4 \%(1,734 / 2,006)$ in the classification of AAH, AIS and MIA. Diagnostic accuracy of FS was shown in Table 2. Multivariate logistic regression model analysis showed that pathological tumor size was the only independent predictive factor of upstage [ $<1 \mathrm{~cm}$ : reference; $1-1.4 \mathrm{~cm}$ : odds ratio (OR) 3.71, 95\% confidence interval (CI): 1.94-11.36, $\mathrm{P}<0.001 ;>1.5 \mathrm{~cm}:$ OR 3.19, 95\% CI: 1.52-14.25; $\mathrm{P}=0.008]$ (Table 3).

AIS and MIA $\geq 1 \mathrm{~cm}$ by FS was more likely upstage to invasive adenocarcinoma compared to those with tumor size $<1 \mathrm{~cm}(\mathrm{P}<0.001)$. Eight invasive adenocarcinomas were found in tumors $\leq 1 \mathrm{~cm}$, two from AIS and six from MIA. Fifty-five invasive adenocarcinomas were found in tumors with a size $>1 \mathrm{~cm}$, seven from AIS and forty-eight from MIA. Our findings demonstrated that AIS or MIA $\geq 1 \mathrm{~cm}$ by FS were more likely upstaged to invasive adenocarcinoma. Hence, FS diagnosis of AIS or MIA should be considered cautiously for tumors $\geq 1 \mathrm{~cm}$ to avoid potentially insufficient resection.

\section{Reasons for the upstage by FP}

Nineteen cases $(7 \%, 19 / 272)$ were classified as low-quality. Example of low-quality FS is shown in Figure $2 A$ (MIA by FS), which influence the interpretation of the presence or extent of invasion. The corresponding permanent section is shown in Figure 2B (invasive adenocarcinoma by FP).

Seventy-eight upstage cases $(28.7 \%, 78 / 272)$ were 
Table 1 Clinicopathologic characteristics and perioperative outcomes

\begin{tabular}{|c|c|c|c|}
\hline \multirow{2}{*}{ Characteristics } & \multicolumn{2}{|c|}{ Final pathology } & \multirow{2}{*}{$\mathrm{P}$} \\
\hline & Invasive adenocarcinoma $(n=63)$ & AAH/AIS/MIA $(n=1,943)$ & \\
\hline Age, years, No. (\%) & & & 0.013 \\
\hline$\geq 60$ & $27(42.9)$ & $554(28.5)$ & \\
\hline Gender, No. (\%) & & & 0.122 \\
\hline Female & $36(57.1)$ & $1,292(66.5)$ & \\
\hline Smoking history, No. (\%) & & & 0.205 \\
\hline Ever/current & $10(15.9)$ & $210(10.8)$ & \\
\hline Never & $53(84.1)$ & 1,733 (89.2) & \\
\hline \multicolumn{4}{|l|}{ Radiologic measurements (on CT) } \\
\hline Whole tumor size, $\mathrm{cm}$ & $1.47 \pm 0.45$ & $0.83 \pm 0.30$ & $<0.001$ \\
\hline Solid component size, $\mathrm{cm}$ & $0.71 \pm 0.46$ & $0.02 \pm 0.10$ & $<0.001$ \\
\hline CTR & $0.48 \pm 0.26$ & $0.02 \pm 0.12$ & $<0.001$ \\
\hline Primary tumor location, No. (\%) & & & 0.022 \\
\hline Upper and middle lobe & $37(58.7)$ & $1,398(71.9)$ & \\
\hline Lower lobe & $26(41.3)$ & $545(28.1)$ & \\
\hline Pathological tumor size, $\mathrm{cm}$ & $1.17 \pm 0.45$ & $0.72 \pm 0.29$ & $<0.001$ \\
\hline Total LN removed & $3.69 \pm 4.85$ & $3.49 \pm 4.63$ & 0.818 \\
\hline No & $6(9.5)$ & $95(4.9)$ & \\
\hline VPI, No. (\%) & & & $<0.001$ \\
\hline Yes & $2(3.2)$ & $0(0)$ & \\
\hline No & $61(96.8)$ & $1,943(100.0)$ & \\
\hline STAS, No. (\%) & & & 0.115 \\
\hline Yes & $1(1.6)$ & $0(0)$ & \\
\hline No & $62(98.4)$ & $1,943(100.0)$ & \\
\hline LN positive, No. (\%) & & & $>0.999$ \\
\hline Yes & $0(0)$ & $0(0)$ & \\
\hline No & $32(100)$ & $492(100.0)$ & \\
\hline Postoperative chemotherapy, No. (\%) & & & $<0.001$ \\
\hline Yes & $7(11.1)$ & $0(0)$ & \\
\hline No & $56(88.9)$ & $1,943(100.0)$ & \\
\hline
\end{tabular}

$\mathrm{AAH}$, atypical adenomatous hyperplasia; AIS, adenocarcinoma in situ; MIA, minimally invasive adenocarcinoma; IA, invasive adenocarcinoma; CT, computed tomography; LN, lymph node; CTR, consolidation-to-tumor ratio; VATS, video-assisted thoracic surgery; VPI, visceral pleural invasion; STAS, tumor spread through air space. 


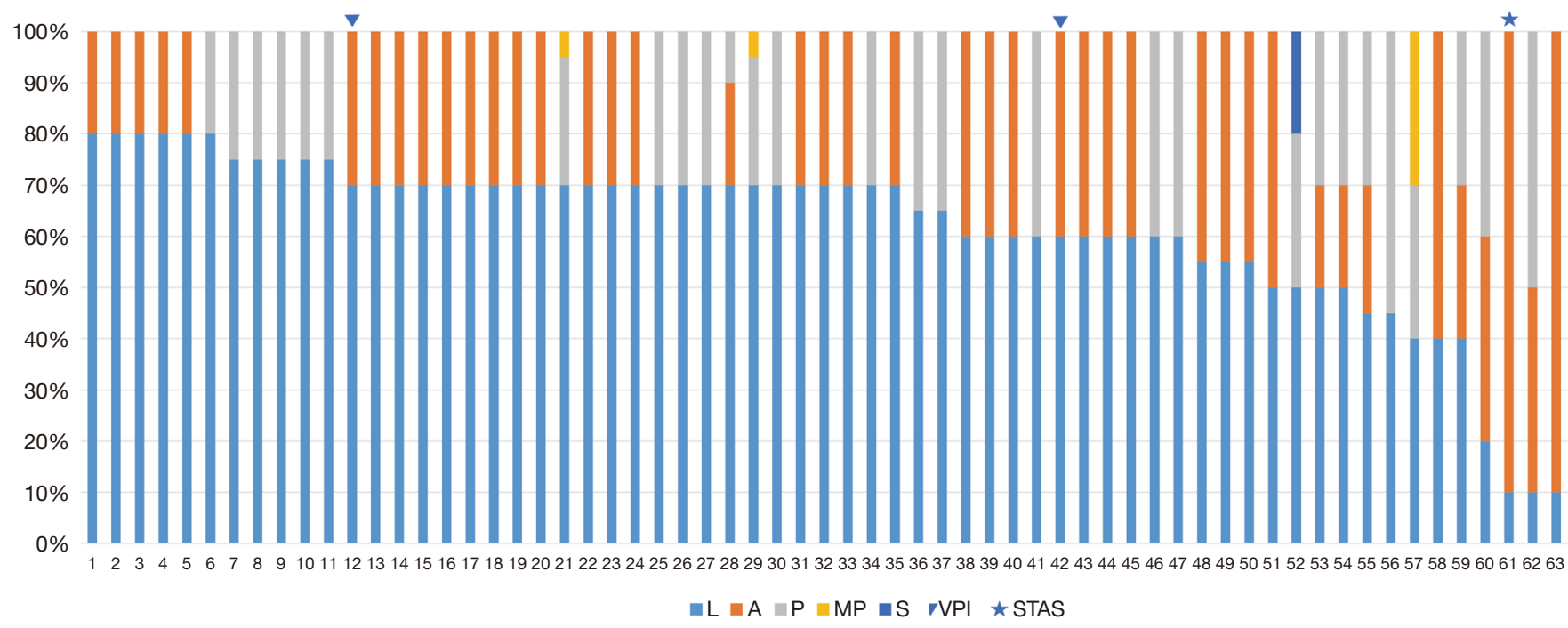

Figure 1 The pathological characteristics of 63 patients upstage to invasive lung adenocarcinoma.

Table 2 Accuracy of the diagnosis of frozen section

\begin{tabular}{|c|c|c|c|c|c|}
\hline Frozen section & \multicolumn{4}{|c|}{ Final pathology } & Total, $\mathrm{N}=2,006$ \\
\hline AAH, No. (\%) & 77 (100.0) & $82(7.9)$ & $0(0)$ & $0(0)$ & 159 \\
\hline AIS, No. (\%) & $0(0)$ & 957 (92.1) & $127(15.4)$ & 9 (14.3) & 1,093 \\
\hline MIA, No. (\%) & $0(0)$ & $0(0)$ & $700(84.6)$ & $54(85.7)$ & 754 \\
\hline Sensitivity, \% & 100 & 92.1 & 84.6 & - & \\
\hline Specificity, \% & 95.7 & 85.9 & 95.4 & - & \\
\hline PPV, \% & 48.4 & 87.7 & 92.8 & - & \\
\hline NPV, \% & 100 & 91 & 89.9 & - & \\
\hline
\end{tabular}

$\mathrm{AAH}$, atypical adenomatous hyperplasia; AIS, adenocarcinoma in situ; MIA, minimally invasive adenocarcinoma; NPV, negative predictive value; PPV, positive predictive value.

attributed to interpretation error, 52 were objective interpretation error, and 26 cases were subjective interpretation error. An example of tumor interstitial fibrosis is shown in Figure $2 C$ (MIA by FS), atrophic alveolar cavities involved in the hyperplastic fibrous tissue, making it difficult to interpret the extend of invasion, and the corresponding permanent section is shown in Figure 2D (invasive adenocarcinoma by FP). Another diagnostic trap occurred when tumor cells fall into the alveolar cavity, which made it difficult to distinguish them from intra-alveolar macrophages (Figure 2E; MIA by FS), and the corresponding permanent section was shown in Figure $2 F$ (invasive adenocarcinoma by FP). On the contrary, intra-alveolar macrophages that fall off into the alveolar cavity may also be misdiagnosed as tumor cells (Figure 2G,H).

One-hundred seventy-five upstage cases $(64.3 \%$, $175 / 272)$ were attributed to sampling error. Because of the large tumor volume, the range of invasion in paraffinembedded tissues may exceed that observed in the FS due to the limitations of FS sampling, and analysis of deeper paraffin-embedded sections can reveal other invasive lesions 
Table 3 Logistic regression model for the predictors of upgrade in final pathology diagnoses

\begin{tabular}{|c|c|c|c|}
\hline Predictors & $\begin{array}{c}\text { Univariable analysis } \\
\text { P value }\end{array}$ & \multicolumn{2}{|c|}{ Multivariable analysis } \\
\hline Solid component size ( $\geq 0.5$ vs. $<0.5 \mathrm{~cm})$ & 0.028 & $1.64(0.87-3.21)$ & 0.079 \\
\hline CTR $(\geq 0.25$ vs. $<0.25)$ & 0.196 & & \\
\hline \multicolumn{4}{|l|}{ Pathological tumor size, $\mathrm{cm}$} \\
\hline $1-1.4$ & $<0.001$ & $3.71(1.94-11.36)$ & $<0.001$ \\
\hline$\geq 1.5$ & $<0.001$ & $3.19(1.52-14.25)$ & 0.008 \\
\hline Preoperative CEA ( $\geq 5$ vs. $<5$ ng/mL) & 0.415 & & \\
\hline
\end{tabular}

Variables with $\mathrm{P}$ value $<0.1$ in univariate models were analyzed in multivariate analysis model. CTR, consolidation-to-tumor ratio; CEA, carcinoembryonic antigen; OR, odds ratio; $\mathrm{Cl}$, confidence interval.

or an expanded range of invasion. As we have demonstrated, AIS and MIA $\geq 1 \mathrm{~cm}$ by FS were more likely upstaged to invasive adenocarcinoma. Four examples of sampling error were shown in Figure 3. In the first example, the case was diagnosed as MIA on FS (Figure $3 A$ ), which was upstaged to lepidic-predominant invasive adenocarcinoma on the permanent section (Figure 3B). In the second example, the case was diagnosed as AIS on FS (Figure 3C), which upstaged to lepidic-predominant invasive adenocarcinoma on permanent section (Figure 3D). In the third example, the case was diagnosed as MIA on FS (Figure $3 E$ ), which was upstaged to invasive adenocarcinoma with visceral pleural invasion (VPI) on the permanent section (Figure $3 F$ ). In the fourth example, the case was diagnosed as AIS on FS (Figure $3 G$ ), which was upstaged to invasive adenocarcinoma with tumor spread through air space (STAS) on the permanent section (Figure 3H).

\section{Clinical influence and complementary treatment of the tumors with FS errors}

Once a tumor was intraoperatively diagnosed as AAH, AIS or MIA, sublobar resection was performed. Among the 272 patients with FS errors, the errors of 209 patients (upstaged to AIS or MIA) had no influence on the clinical therapy strategy because the extent of their resections were enough. Of the 63 cases with invasive adenocarcinomas, 28 patients with lepidic predominant adenocarcinoma underwent wedge resection and the remaining 35 patients underwent segmentectomy. After informed consent, only five patients agreed to undergo a second operation for completion lobectomy, and another seven patients agreed to received chemotherapy. The remaining 51 patients were followed up closely after sublobar resection.

Complete lobectomy and systematic lymph node dissection were performed 3-4 weeks later after the first operation. No satellite lesions were found in the lung specimen, and all of the lymph nodes were negative in the pathological examination. Seven patients received two cycles postoperative chemotherapy 4 weeks later after the first operation. The chemotherapy regimen consisted of platinum plus pemetrexed. The information of complementary treatment was shown in Table S2.

\section{The prognosis of patients with pathological upstage}

Two invasive adenocarcinoma cases without complementary treatment experienced a local recurrence. One recurrent case was lepidic-predominant with VPI positive treated by segmentectomy with a tumor size of $1.5 \mathrm{~cm}$. The recurrence site was the ipsilateral hilum. Another recurrent case was acinar-predominant with STAS positive treated by wedge resection with a tumor size of $1.2 \mathrm{~cm}$. The recurrence site was the resection margin. Two recurrent cases were identified by thin-slice CT and positron emission/computed tomography (PET/CT) and did not undergo needle aspiration cytology examination. The possibility of a second primary lung cancer was ruled out by a multidisciplinary team. Both two patients received chemotherapy because they did not harbor driving mutations, such as epidermal growth factor receptor (EGFR) mutation. The 5-year RFS rate of patients with invasive adenocarcinoma without complementary treatment was $96.1 \%$ (49/51), whereas the five-year RFS rate of those AAH/AIS/MIA and invasive 
Frozen sections
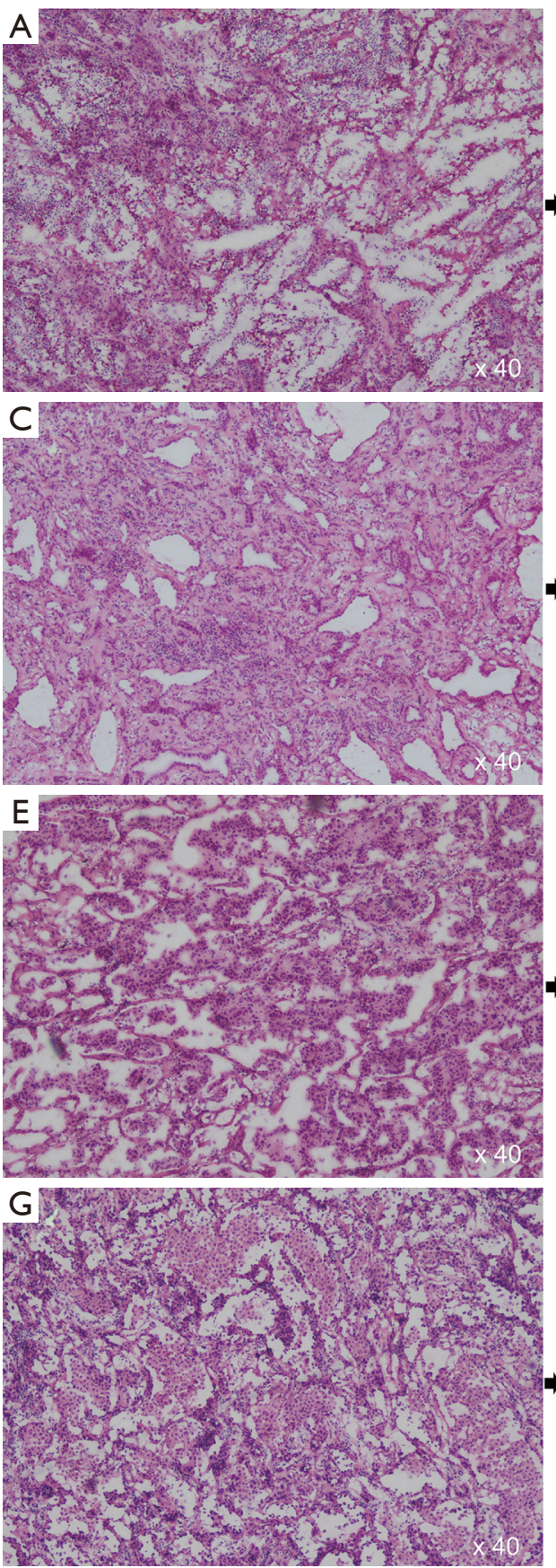

Permanent sections
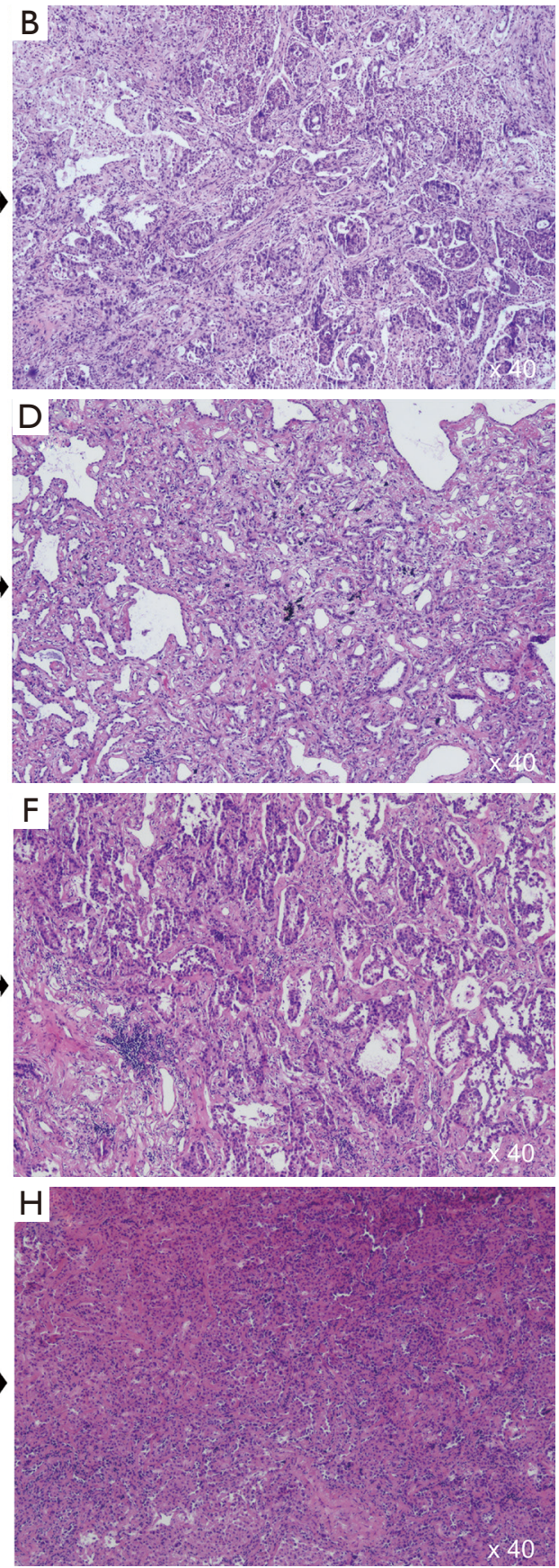

Figure 2 Examples of low-quality FS and interpretation errors. (A) Example of low-quality FS (MIA by FS), which influence the interpretation of the extent of invasion $(\mathrm{H} \& \mathrm{E} \times 40)$, (B) the corresponding permanent section (invasive adenocarcinoma by $\mathrm{FP})(\mathrm{H} \& \mathrm{E} \times 40)$. (C) Example of tumor interstitial fibrosis (MIA by FS) (H\&E $\times 40)$, atrophic alveolar cavities involved in the hyperplastic fibrous tissue, making it difficult to interpret the extend of invasion, (D) the corresponding permanent section (invasive adenocarcinoma by FP) (H\&E $\times 40$ ). (E) Tumor cells fall off into the alveolar cavity, which is difficult to distinguish from intra-alveolar macrophages (MIA by FS) (H\&E $\times 40)$, (F) the corresponding permanent section (invasive adenocarcinoma by FP) $(\mathrm{H} \& \mathrm{E} \times 40)$. (G) Intra-alveolar macrophages fall off into the alveolar cavity may also be misdiagnosed as tumor cells $(\mathrm{H} \& \mathrm{E} \times 40),(\mathrm{H})$ the corresponding permanent section $(\mathrm{H} \& \mathrm{E} \times 40)$. MIA, minimally invasive adenocarcinoma; FS, frozen section; FP, final pathology; H\&E, hematoxylin-eosin staining. 
Frozen sections
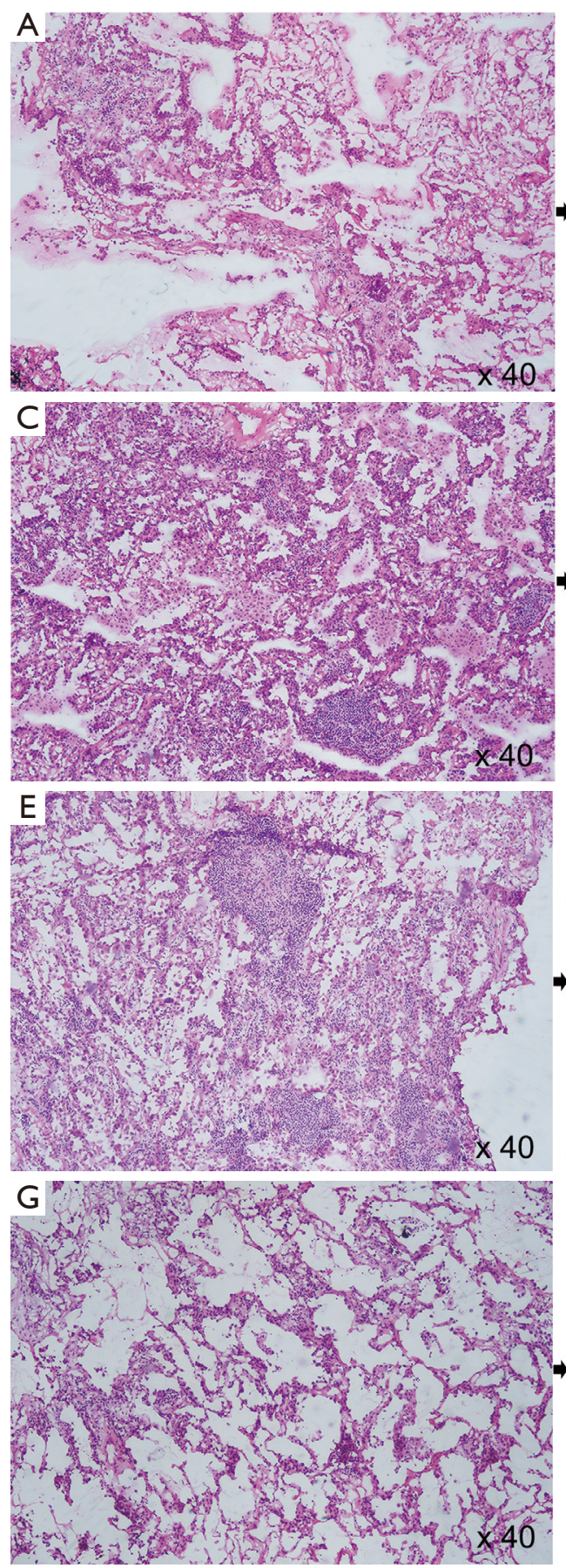

Permanent sections
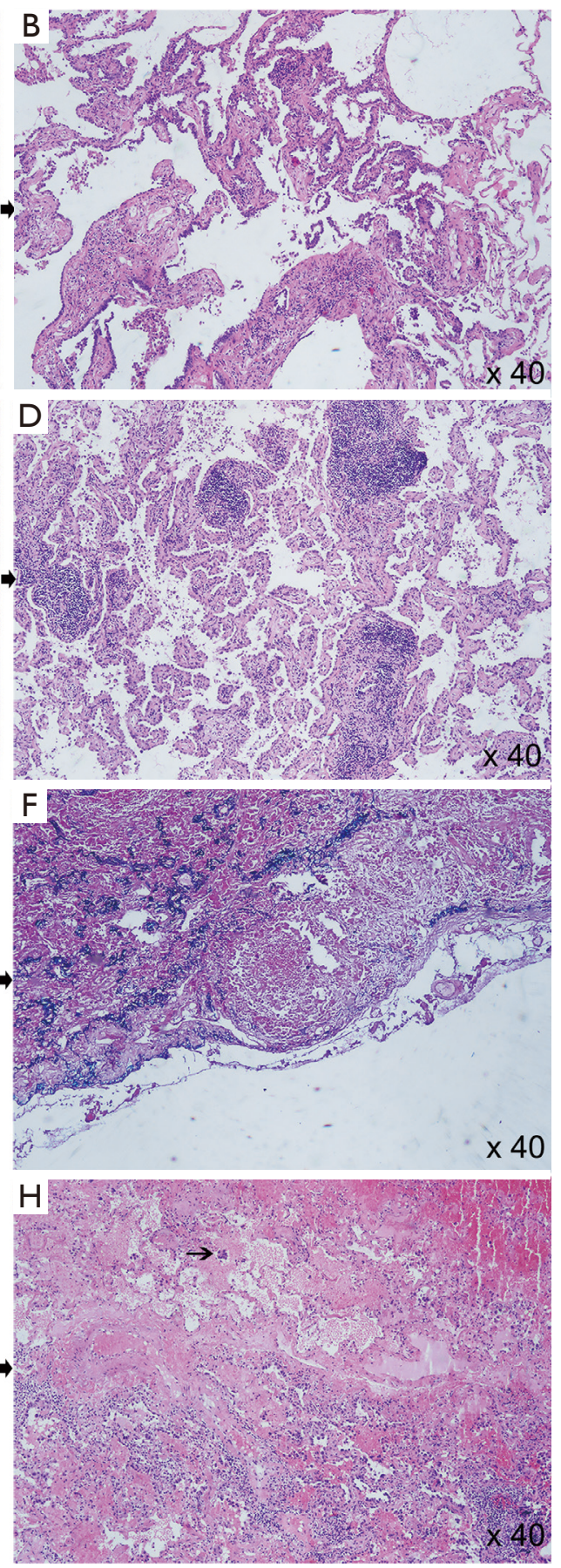

Figure 3 Examples of sampling errors. Tumor was diagnosed as MIA on FS (A), which upstaged to lepidic-predominant invasive adenocarcinoma on permanent section (B) (H\&E $\times 40)$. Tumor was diagnosed as AIS on FS (C), which upstaged to lepidic-predominant invasive adenocarcinoma on permanent section (D) $(\mathrm{H} \& \mathrm{E} \times 40)$. Tumor was diagnosed as MIA on FS (E), which upstaged to invasive adenocarcinoma with visceral pleural invasion on permanent section (F) (H\&E ×40). Tumor was diagnosed as AIS on FS (G), which upstaged to invasive adenocarcinoma with STAS on permanent section $(\mathrm{H})(\mathrm{H} \& \mathrm{E} \times 40)$. MIA, minimally invasive adenocarcinoma; FS, frozen section; STAS, tumor spread through air space; H\&E, hematoxylin-eosin staining. 
adenocarcinoma with complementary treatment was $100 \%$. No patient died until December 01, 2019.

\section{Discussion}

Today, lung cancer represents the leading cause of cancerrelated mortality worldwide in addition to in China $(20,21)$. Clinically, AAH/AIS/MIA only needs sublobar resection, whereas invasive adenocarcinomas need a lobectomy for a potential cure. Hence, it is very important to distinguish between AAH/AIS/MIA and invasive adenocarcinoma to the surgeons in frozen pathology. However, pathological upstages occur for various reasons (13). No previous researchers have considered the need to perform complementary lobectomies for those patients who have had pathological upstages and already underwent sublobar resections. Our findings indicate that complementary treatment is encouraged in AIS/MIA upstaged to invasive adenocarcinoma by FP after sublobar resection to avoid recurrence. Further randomized controlled trials (RCTs) are needed to verify the results of our study.

Treatment strategies are largely designed according to radiological features. Previous studies have demonstrated that the consolidation-to-tumor ratio (CTR) is associated with patient prognosis $(16,22)$. The CTR determined from preoperative CT images could be used to predict tumor invasion and help surgeons choose the most effective surgical strategies (23). With regard to radiological features, the whole tumor size, consolidation size, and CTR based on high-resolution computed tomography (HRCT) are widely used clinically (24-26). Saji et al. evaluated differences between the whole tumor and the solid component size for predicting malignant grade and prognosis (27). The authors found the solid component size to be superior in predicting pathological higher-grade tumors and prognoses. In our study, the CT feature measurement values for patients who had had pathological upstages were larger and there were significant differences between patients with and without pathological upstage (solid component size: $\mathrm{P}<0.001$; whole tumor size: $\mathrm{P}<0.001$; CTR: $\mathrm{P}<0.001)$.

FS has been proven to enable the precise identification of histological patterns. Previously, Marchevsky et al. (28) demonstrated intraoperative FS to be a sensitive and specific procedure for the diagnosis of malignancy from small solitary pulmonary nodules. Since the 2011 publication of the new IASLC/ATS/ERS classification, some researchers have evaluated the diagnostic accuracy of intraoperative FS according to this new classification $(12,29)$. In this study, we found that AIS and MIA $\geq 1 \mathrm{~cm}$ by FS were more likely upstage to invasive adenocarcinoma because of sampling error. For pathologists, FS diagnosis and tumor size should be considered jointly to predict the final pathological diagnosis; for thoracic surgeons, FS diagnosis of AIS and MIA should be performed cautiously for tumors with size $\geq 1 \mathrm{~cm}$ to avoid insufficient resection.

Walts et al. compared the results of FS with final pathologies, and reported $97 \%$ of the FS to be correct for invasive adenocarcinoma, whereas only $59 \%$ and $46 \%$ were correct for AIS and MIA, respectively (29). The authors identified the most common reason for diagnostic error to be over-diagnosis. Yeh et al. (12) also used intraoperative FS to identify histological patterns in stage I lung adenocarcinomas of $\leq 3 \mathrm{~cm}$, and found the accuracy of MIA to be unsatisfactory (41.3\%). In their study, nearly half of the interpretations were over-diagnoses of invasive adenocarcinoma. The sampling numbers of the above two studies were relatively small, which could have resulted in some bias and a decrease in the diagnostic accuracy of the intraoperative FS. Liu et al. (17) recommended that in intraoperative FS, given the intractability of judging invasion, it is better for pathologists to be more conservative and thereby achieve higher precision in their diagnosis rates in addition to adopting improved methods, such as inflation and elastic stain.

With respect to surgical procedure, the efficacy of sublobar resection has been a matter of some doubt (30). The only RCT in this regard was conducted in 1995 , in which the researchers reported that in patients with T1N0M0 non-small cell lung cancer (NSCLC), sublobar resection could not improve preoperative morbidity, mortality, or postoperative pulmonary function when compared with lobectomy; they recommended lobectomy as the standard treatment (31). The result of many studies supports the conclusions of this RCT $(32,33)$. By contrast, many researchers have observed that sublobar resection is not inferior to lobectomy in patients with small, localized stage IA NSCLC, whether the patients are elderly or young (34-36). However, in patients with AIS and MIA, sublobar resection has proven to be a valid oncological procedure (6). In our study, patients with low-risk histological grades (from AAH/AIS to MIA) all had excellent prognoses (five-year RFS: $100 \%$; five-year OS: $100 \%$ ) and were not required to undergo a complementary treatment. However, in patients with invasive lung adenocarcinoma, sublobar resection may not be enough. Of them, two patients experienced a local recurrence after sublobar resection without complementary 
lobectomy.

There are some limitations in our study. First, it was a retrospective study and the nature of retrospective analysis may lead to selection bias. Also, all the data were extracted from a single center, which may result in a relative lack of generalization and representativeness. Second, although 2,006 patients were enrolled in our study, the sample size of patients with histological upstages was small. Third, although the median follow-up time was 52 months, with relatively early-stage adenocarcinoma, occurrences of relapse or even cancer-specific death need more time. Therefore, the follow-up time was insufficient. Fourth, two recurrent patients did not undergo pathological examination to rule out the possibility of a secondary primary tumor. Further prospective studies are necessary to address these issues.

\section{Conclusions}

Pathologists should be more cautious about AIS and MIA with tumor size $\geq 1 \mathrm{~cm}$ to avoid underestimation and potentially insufficient resection. Complementary treatment is encouraged in AIS/MIA upstaged to invasive adenocarcinoma by $\mathrm{FP}$ after sublobar resection to avoid local recurrence.

\section{Acknowledgments}

Funding: This work was supported by the Clinical Research Project of Shanghai Pulmonary Hospital (fk18001), the projects from the Fundamental Research Funds for the Central Universities (22120180607), the National Natural Science Foundation of China (81802256), Science and Technology Commission of Shanghai Municipality (15411968400 and 14411962600), the "Chen Guang" project supported by Shanghai Municipal Education Commission and Shanghai Education Development Foundation (18CG19).

\section{Footnote}

Reporting Checklist: The authors have completed the TRIPOD reporting checklist. Available at http://dx.doi. org/10.21037/tlcr-20-828

Data Sharing Statement: Available at http://dx.doi. org/10.21037/tlcr-20-828
Peer Review File: Available at http://dx.doi.org/10.21037/ tlcr-20-828

Conflicts of Interest: All authors have completed the ICMJE uniform disclosure form (available at http://dx.doi. org/10.21037/tlcr-20-828). The authors have no conflicts of interest to declare.

Ethical Statement: The authors are accountable for all aspects of the work in ensuring that questions related to the accuracy or integrity of any part of the work are appropriately investigated and resolved. All procedures performed in this study were in accordance with the Declaration of Helsinki (as revised in 2013) and approved by the Institutional Review Board of the Shanghai Pulmonary Hospital (IRB, K20-432). Because of the retrospective nature of the research, the requirement for informed consent was waived.

Open Access Statement: This is an Open Access article distributed in accordance with the Creative Commons Attribution-NonCommercial-NoDerivs 4.0 International License (CC BY-NC-ND 4.0), which permits the noncommercial replication and distribution of the article with the strict proviso that no changes or edits are made and the original work is properly cited (including links to both the formal publication through the relevant DOI and the license). See: https://creativecommons.org/licenses/by-nc-nd/4.0/.

\section{References}

1. Jemal A, Bray F, Center MM, et al. Global cancer statistics. CA Cancer J Clin 2011;61:69-90.

2. Travis WD, Brambilla E, Noguchi M, et al. International association for the study of lung cancer/american thoracic society/european respiratory society international multidisciplinary classification of lung adenocarcinoma. J Thorac Oncol 2011;6:244-85.

3. National Comprehensive Cancer Network. NCCN clinical practice guidelines in oncology (NCCN Guidelines): non-small cell lung cancer, version 1. 2020. Available online: https://www.nccn.org/patients/guidelines/cancers. aspx\#nsclc

4. Blasberg JD, Pass HI, Donington JS. Sublobar resection: a movement from the Lung Cancer Study Group. J Thorac Oncol 2010;5:1583-93.

5. Varlotto JM, Medford-Davis LN, Recht A, et al. 
Identification of stage I non-small cell lung cancer patients at high risk for local recurrence following sublobar resection. Chest 2013;143:1365-77.

6. Russell PA, Wainer Z, Wright GM, et al. Does lung adenocarcinoma subtype predict patient survival?: A clinicopathologic study based on the new International Association for the Study of Lung Cancer/American Thoracic Society/European Respiratory Society international multidisciplinary lung adenocarcinoma classification. J Thorac Oncol 2011;6:1496-504.

7. Dai C, Shen J, Ren Y, et al. Choice of Surgical Procedure for Patients With Non-Small-Cell Lung Cancer $</=1 \mathrm{~cm}$ or $>1$ to $2 \mathrm{~cm}$ Among Lobectomy, Segmentectomy, and Wedge Resection: A Population-Based Study. J Clin Oncol 2016;34:3175-82.

8. Hattori A, Matsunaga T, Hayashi T, et al. Prognostic Impact of the Findings on Thin-Section Computed Tomography in Patients with Subcentimeter Non-Small Cell Lung Cancer. J Thorac Oncol 2017;12:954-62.

9. Kadota K, Villena-Vargas J, Yoshizawa A, et al. Prognostic significance of adenocarcinoma in situ, minimally invasive adenocarcinoma, and nonmucinous lepidic predominant invasive adenocarcinoma of the lung in patients with stage I disease. Am J Surg Pathol 2014;38:448-60.

10. Kocaturk CI, Gunluoglu MZ, Cansever L, et al. Survival and prognostic factors in surgically resected synchronous multiple primary lung cancers. Eur J Cardiothorac Surg 2011;39:160-6.

11. He P, Yao G, Guan Y, et al. Diagnosis of lung adenocarcinoma in situ and minimally invasive adenocarcinoma from intraoperative frozen sections: an analysis of 136 cases. J Clin Pathol 2016;69:1076-80.

12. Yeh YC, Nitadori J, Kadota K, et al. Using frozen section to identify histological patterns in stage I lung adenocarcinoma of $</=3 \mathrm{~cm}$ : accuracy and interobserver agreement. Histopathology 2015;66:922-38.

13. Zhu E, Xie H, Dai C, et al. Intraoperatively measured tumor size and frozen section results should be considered jointly to predict the final pathology for lung adenocarcinoma. Mod Pathol 2018;31:1391-9.

14. Koike T, Koike T, Yoshiya K, et al. Risk factor analysis of locoregional recurrence after sublobar resection in patients with clinical stage IA non-small cell lung cancer. J Thorac Cardiovasc Surg 2013;146:372-8.

15. Goldstraw P, Chansky K, Crowley J, et al. The IASLC Lung Cancer Staging Project: Proposals for Revision of the TNM Stage Groupings in the Forthcoming (Eighth) Edition of the TNM Classification for Lung Cancer. J
Thorac Oncol 2016;11:39-51.

16. Asamura H, Hishida T, Suzuki K, et al. Radiographically determined noninvasive adenocarcinoma of the lung: survival outcomes of Japan Clinical Oncology Group 0201. J Thorac Cardiovasc Surg 2013;146:24-30.

17. Liu S, Wang R, Zhang Y, et al. Precise Diagnosis of Intraoperative Frozen Section Is an Effective Method to Guide Resection Strategy for Peripheral Small-Sized Lung Adenocarcinoma. J Clin Oncol 2016;34:307-13.

18. Suzuki K, Koike T, Asakawa T, et al. A prospective radiological study of thin-section computed tomography to predict pathological noninvasiveness in peripheral clinical IA lung cancer (Japan Clinical Oncology Group 0201). J Thorac Oncol 2011;6:751-6.

19. Tsutani Y, Miyata Y, Nakayama H, et al. Sublobar resection for lung adenocarcinoma meeting nodenegative criteria on preoperative imaging. Ann Thorac Surg 2014;97:1701-7.

20. Chen $W$, Zheng R, Baade PD, et al. Cancer statistics in China, 2015. CA Cancer J Clin 2016;66:115-32.

21. Siegel R, Naishadham D, Jemal A. Cancer statistics, 2013. CA Cancer J Clin 2013;63:11-30.

22. Hattori A, Matsunaga T, Takamochi K, et al. Importance of Ground Glass Opacity Component in Clinical Stage IA Radiologic Invasive Lung Cancer. Ann Thorac Surg 2017;104:313-20.

23. Aokage K, Yoshida J, Hishida T, et al. Limited resection for early-stage non-small cell lung cancer as functionpreserving radical surgery: a review. Jpn J Clin Oncol 2017;47:7-11.

24. Flores RM, Nicastri D, Bauer T, et al. Computed Tomography Screening for Lung Cancer: Mediastinal Lymph Node Resection in Stage IA Nonsmall Cell Lung Cancer Manifesting as Subsolid and Solid Nodules. Ann Surg 2017;265:1025-33.

25. Kudo Y, Matsubayashi J, Saji H, et al. Association between high-resolution computed tomography findings and the IASLC/ATS/ERS classification of small lung adenocarcinomas in Japanese patients. Lung Cancer 2015;90:47-54.

26. Matsuguma H, Oki I, Nakahara R, et al. Comparison of three measurements on computed tomography for the prediction of less invasiveness in patients with clinical stage I non-small cell lung cancer. Ann Thorac Surg 2013;95:1878-84.

27. Saji H, Matsubayashi J, Akata S, et al. Correlation between whole tumor size and solid component size on highresolution computed tomography in the prediction of 
the degree of pathologic malignancy and the prognostic outcome in primary lung adenocarcinoma. Acta Radiol 2015;56:1187-95.

28. Marchevsky AM, Changsri C, Gupta I, et al. Frozen section diagnoses of small pulmonary nodules: accuracy and clinical implications. Ann Thorac Surg 2004;78:1755-9.

29. Walts AE, Marchevsky AM. Root cause analysis of problems in the frozen section diagnosis of in situ, minimally invasive, and invasive adenocarcinoma of the lung. Arch Pathol Lab Med 2012;136:1515-21.

30. Sihoe AD, Van Schil P. Non-small cell lung cancer: when to offer sublobar resection. Lung Cancer 2014;86:115-20.

31. Ginsberg RJ, Rubinstein LV. Randomized trial of lobectomy versus limited resection for T1 N0 non-small cell lung cancer. Lung Cancer Study Group. Ann Thorac Surg 1995;60:615-22; discussion 622-3.

32. Kraev A, Rassias D, Vetto J, et al. Wedge resection vs

Cite this article as: $\mathrm{Su} \mathrm{H}, \mathrm{Gu}$ C, She Y, Xu L, Yang P, Xie H, Zhao S, Wu C, Xie D, Chen C. Predictors of upstage and treatment strategies for stage IA lung cancers after sublobar resection for adenocarcinoma in situ and minimally invasive adenocarcinoma. Transl Lung Cancer Res 2021;10(1):32-44. doi: $10.21037 /$ tlcr-20-828 lobectomy: 10-year survival in stage I primary lung cancer. Chest 2007;131:136-40.

33. Veluswamy RR, Ezer N, Mhango G, et al. Limited Resection Versus Lobectomy for Older Patients With Early-Stage Lung Cancer: Impact of Histology. J Clin Oncol 2015;33:3447-53.

34. Altorki NK, Yip R, Hanaoka T, et al. Sublobar resection is equivalent to lobectomy for clinical stage 1A lung cancer in solid nodules. J Thorac Cardiovasc Surg 2014;147:75462; discussion 762-4.

35. Gu C, Wang R, Pan X, et al. Sublobar resection versus lobectomy in patients aged $</=35$ years with stage IA nonsmall cell lung cancer: a SEER database analysis. J Cancer Res Clin Oncol 2017;143:2375-82.

36. Schuchert MJ, Kilic A, Pennathur A, et al. Oncologic outcomes after surgical resection of subcentimeter nonsmall cell lung cancer. Ann Thorac Surg 2011;91:1681-7; discussion 1687-8. 


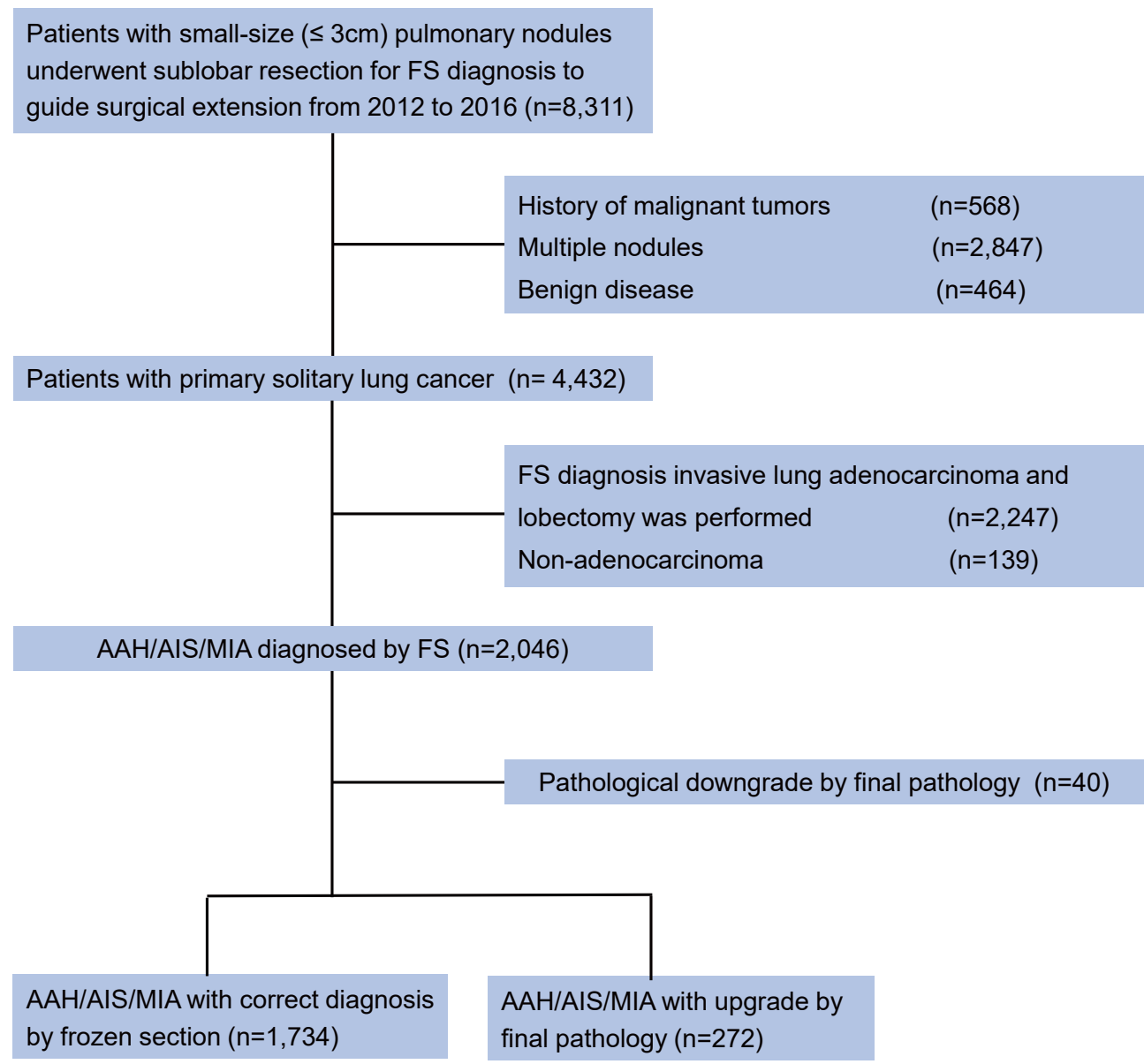

Figure S1 The flowchart of the patient's selection. A total of 8,311 patients with pulmonary nodules who underwent sublobar resection for frozen section (FS) diagnosis to guide surgical extension were identified from January 2012 to December 2016. After exclusion, 2006 patients were included in this study. 
Table S1 The comparison of clinicopathological features between invasive adenocarcinomas with correct diagnosis by FS and 63 invasive adenocarcinomas with underestimation by FS

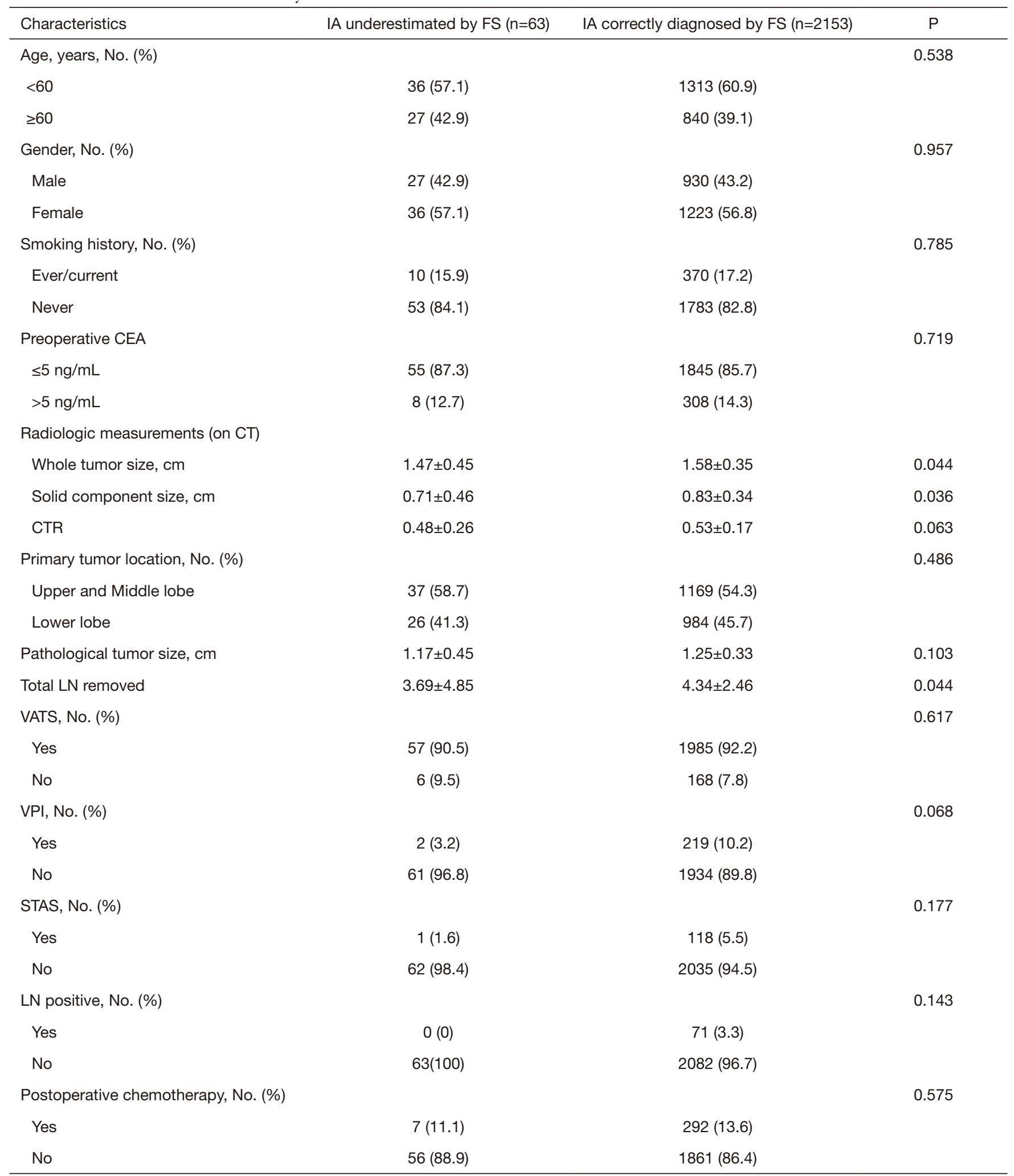

IA, invasive adenocarcinoma; CT, computed tomography; LN, lymph node; CTR, consolidation-to-tumor ratio; VATS, video-assisted thoracic surgery; VPI, visceral pleural invasion; STAS, tumor spread through air space. 
Table S2 The characteristics and outcomes of thirteen patients received complementary treatment

\begin{tabular}{|c|c|c|c|c|c|c|c|c|}
\hline Patients & Age & Gender & $\begin{array}{l}\text { FS } \\
\text { diagnosis }\end{array}$ & $\begin{array}{l}\text { Tumor size, } \\
\text { pathological }\end{array}$ & $\begin{array}{l}\text { Pathological } \\
\text { characteristics }\end{array}$ & Surgical procedure & Complementary treatment & Recurrence \\
\hline 1 & 56 & Male & MIA & $1.3 \mathrm{~cm}$ & Lepidic-pre, MP (+) & Segmentectomy & $\begin{array}{l}\text { Lobectomy and systematic } \\
\text { LN dissection }\end{array}$ & No \\
\hline 2 & 60 & Male & AIS & $1.1 \mathrm{~cm}$ & Lepidic-pre & Wedge resection & $\begin{array}{l}\text { Lobectomy and systematic } \\
\text { LN dissection }\end{array}$ & No \\
\hline 4 & 64 & Male & MIA & $0.9 \mathrm{~cm}$ & Lepidic-pre & Segmentectomy & $\begin{array}{l}\text { Lobectomy and systematic } \\
\text { LN dissection }\end{array}$ & No \\
\hline 5 & 63 & Female & MIA & $1.2 \mathrm{~cm}$ & Acinar-pre; MP (+) & Wedge resection & $\begin{array}{l}\text { Lobectomy and systematic } \\
\text { LN dissection }\end{array}$ & No \\
\hline 7 & 61 & Female & MIA & $1.4 \mathrm{~cm}$ & Papillary-pre; VPI (+) & Segmentectomy & $\begin{array}{l}\text { Two cycles chemotherapy, } \\
\text { carboplatin combined with } \\
\text { pemetrexed }\end{array}$ & No \\
\hline 8 & 59 & Male & MIA & $1.2 \mathrm{~cm}$ & Lepidic-pre & Wedge resection & $\begin{array}{l}\text { Two cycles chemotherapy, } \\
\text { carboplatin combined with } \\
\text { pemetrexed }\end{array}$ & No \\
\hline 9 & 56 & Male & MIA & $1.3 \mathrm{~cm}$ & Lepidic-pre; & Wedge resection & $\begin{array}{l}\text { Two cycles chemotherapy, } \\
\text { carboplatin combined with } \\
\text { pemetrexed }\end{array}$ & No \\
\hline 13 & 52 & Male & MIA & $1.3 \mathrm{~cm}$ & Lepidic-pre & Segmentectomy & $\begin{array}{l}\text { Two cycles chemotherapy, } \\
\text { carboplatin combined with } \\
\text { vinorelbine }\end{array}$ & No \\
\hline
\end{tabular}

AIS, adenocarcinoma in situ; MIA, minimally invasive adenocarcinoma; pre, predominant; LN, lymph node; MP, micropapillary; VPI, visceral pleural invasion; pre, predominant. 